\title{
Scolex morphology of monozoic tapeworms (Caryophyllidea) from the Nearctic Region: taxonomic and evolutionary implications
}

\author{
Mikuláš Oros ${ }^{1}$, Dalibor Uhrovič ${ }^{1}$, Anindo Choudhury ${ }^{2}$, John S. Mackiewicz ${ }^{3}$ and Tomáš Scholz ${ }^{4}$ \\ ${ }^{1}$ Institute of Parasitology, Slovak Academy of Sciences, Košice, Slovakia; \\ ${ }^{2}$ Division of Natural Sciences, St. Norbert College, De Pere, Wisconsin, USA; \\ ${ }^{3}$ Biological Sciences, State University of New York at Albany, New York, USA; \\ ${ }^{4}$ Institute of Parasitology, Biology Centre of the Czech Academy of Sciences, České Budějovice, Czech Republic
}

\begin{abstract}
A comparative study of the scoleces of monozoic tapeworms (Cestoda: Caryophyllidea), parasites of catostomid and cyprinid fishes (Teleostei: Cypriniformes) in the Nearctic Region, was carried out using light and scanning electron microscopy. Scoleces of 22 genera of North American caryophyllideans were characterised and their importance for taxonomy, classification and phylogenetic studies was critically reviewed. Nearctic genera exhibit a much higher variation in the shape and form of scoleces compared with taxa in other biogeographical regions. The following basic scolex types can be recognised in Nearctic caryophyllideans: monobothriate (Promonobothrium Mackiewicz, 1968), loculotruncate (Promonobothrium, Dieffluvium Williams, 1978), bothrioloculodiscate (Archigetes Leuckart, 1878, Janiszewskella Mackiewicz et Deutsch, 1976, Penarchigetes Mackiewicz, 1969, Pseudoglaridacris Oros, Uhrovič et Scholz, 2018), fixomegabothriate (Capingens Hunter, 1927), bulbate and bulboacuminate (Atractolytocestus Anthony, 1958), cuneiloculate (Hypocaryophyllaeus Hunter, 1927, Rowardleus Mackiewicz et Deutsch, 1976, Spartoides Hunter, 1929), biacetabulate, bulboloculate, bothrioloculodiscate (Biacetabulum Hunter, 1927), tholate (Hunterella Mackiewicz et McCrae, 1962), cuneifimbriate (Khawia Hsü, 1935), cuneiform (Calentinella Mackiewicz, 1974, Caryophyllaeides Nybelin, 1922, Edlintonia Mackiewicz, 1970), hastate (Pseudolytocestus Hunter, 1929), loculotholate (Bialovarium Fischthal, 1953, Pliovitellaria Fischthal, 1951), and cuneiformoloculate (Glaridacris Cooper, 1920, Isoglaridacris Mackiewicz, 1965). The same type of scolex may be shared by species of different genera or families and species of the same genus can have a scolex of conspicuously different morphology, e.g. in Promonobothrium. Scolex morphology may be therefore of limited use in generic designation.
\end{abstract}

Key words: Cestoda, scolex terminology, fish, Catostomidae, comparative morphology, scanning electron microscopy, identification, North America

The scolex of tapeworms plays a key role in their attachment in the intestinal lumen of the definitive host and is the site of the most intimate interface between the parasite and its host (Smyth and McManus 1988). Scolex morphology is thus a result of long-term adaptation of the parasite to its host. Because of conspicuous differences between tapeworms from different groups of vertebrate hosts, scolex morphology has been used as one of the key characters for a higher-level (ordinal) classification of tapeworms (Schmidt 1986, Khalil et al. 1994) and it is also widely used for taxonomy at the generic level (Schmidt 1986, Caira and Jensen 2017).

In caryophyllidean tapeworms, which represent one of the dominant groups of intestinal parasites of cypriniform and siluriform fishes (Scholz and Kuchta 2017), scolex morphology has been used to characterise individual gen- era (Mackiewicz 1994), but also to distinguish congeneric species (Ash et al. 2011a). No single scolex type characterises caryophyllideans (Mackiewicz 1970). Oros et al. (2010) studied scolex morphology of Palaearctic species and found it to represent a useful tool for species identification. However, studies on the species of Caryophyllaeus Gmelin, 1790 in the Palaearctic Region have revealed morphological plasticity of scolex shape in tapeworms from different hosts, casting doubts upon general suitability of scolex morphology in caryophyllidean taxonomy (Barčák et al. 2014, 2017, Hanzelová et al. 2015).

In North America, caryophyllideans are the dominant group of cestodes in catostomid and, to a much lesser extent, cyprinid fishes, being represented by 55 species in 22 genera (Mackiewicz 1972, Hoffman 1999, Scholz and Oros 2017). They represent $56 \%$ of all species of adult cestodes 
A

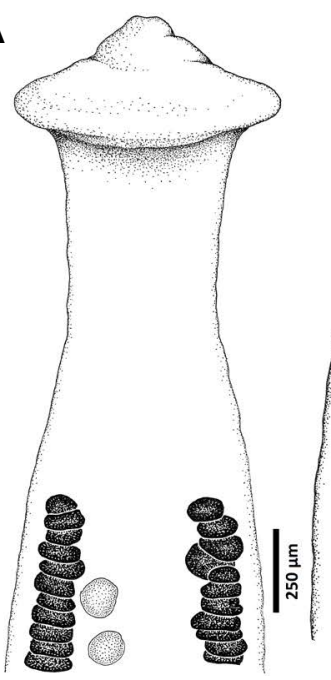

B

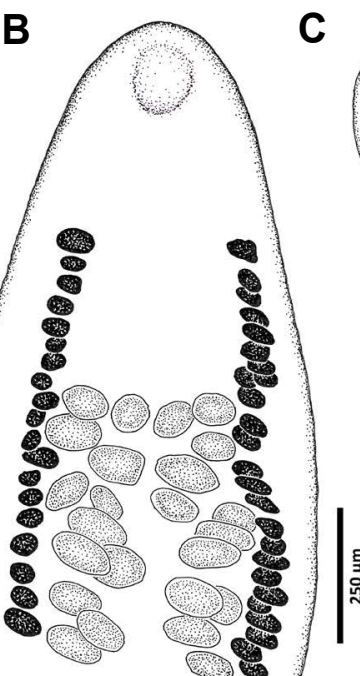

C

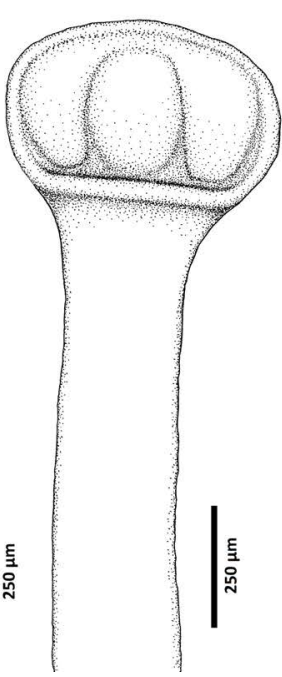

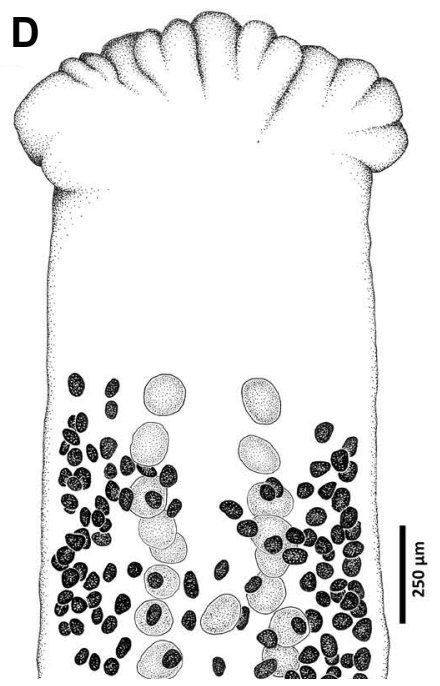

Fig. 1. Line drawings of the anterior parts of the Nearctic species of the Caryophyllidea with the anteriormost vitelline follicles (black) and testes (stippled). A - Atractolytocestus huronensis Anthony, 1958 ex Cyprinus carpio Linnaeus; B - Bialovarium nocomis Fischthal, 1953 ex Nocomis biguttatus (Kirtland) (redrawn from Fischthal 1954); C - Homeomorpha mackiewiczi Dutton et Barger, 2014 ex Minytrema melanops (Rafinesque) (redrawn from Dutton and Barger 2014); D - Khawia japonensis (Yamaguti, 1934) ex Cyprinus carpio.

in freshwater fishes of the Nearctic Region (Scholz and Kuchta 2017). Except for three genera, namely Biacetabulum Hunter, 1927, Isoglaridacris Mackiewicz, 1965 (both genera with 10 spp. each) and Promonobothrium Mackiewicz, 1968 (9 spp.), Nearctic genera are species-poor and 12 genera are monotypic (Scholz and Oros 2017).

Most of these genera are distinguished from each other by characteristics of the reproductive system (see the keys in Mackiewicz 1994), but scolex morphology was proposed as a useful tool for classification and identification of North American species by numerous authors (e.g., Hunter 1930, McCrae 1962, Mackiewicz 1970, 1972, 1994, Mackiewicz and McCrae 1965, Williams 1977). Hayunga and Mackiewicz (1988) studied histology of the scolex and neck region of Glaridacris laruei (Lamont, 1921) (= Pseudoglaridacris laruei) and Glaridacris catostomi Cooper, 1920, and Hayunga (1979) studied the intestinal pathology caused by three species of North American caryophyllideans to their host, the white sucker, Catostomus commersonii (Lacépède). However, a detailed comparative study on the scolex morphology of Nearctic caryophyllideans based on both light and scanning electron microscopy had not been carried out.

As part of a collaborative effort to better characterise the global diversity of cestodes (Caira and Jensen 2017, Scholz and Kuchta 2017), scolex morphology of caryophyllideans parasitising North American freshwater fishes was critically reviewed in this study using light and scanning electron microscopy. Whenever possible, material newly collected by the present authors was processed using the same method (heat-fixation) to ensure comparability of morphological and biometrical data.

\section{MATERIALS AND METHODS}

Newly collected specimens of 31 species (see below for the list of species) were processed for light (LM) and scanning elec- tron microscopical (SEM) observations. Methods of processing were the same as previously reported in detail (Oros et al. 2010, 2016, Scholz et al. 2015). Briefly, worms were removed from the intestine of freshly killed fish host, rinsed in saline, fixed with hot (almost boiling) 4\% neutral buffered formaldehyde solution (= $10 \%$ buffered formalin). For light microscopical examination, specimens were stained with carmine, dehydrated in a graded ethanol series, cleared in clove oil, and mounted in Canada balsam. Drawings were made using a drawing attachment on a Leica DM 5000B (Leica Microsystems, Wetzlar, Germany) light microscope. For histological study specimens were embedded in paraffin wax, sectioned at $12-15 \mu \mathrm{m}$ thickness, and stained with Weigert's haematoxylin.

For SEM studies, formalin-fixed specimens were dehydrated through a graded ethanol series, followed by a graded amylacetate series, dried by HMDS (hexamethyldisilazane), sputter-coated with 20-25 nm of gold and examined with JEOL JSEM 6510LV microscope (JEOL Ltd., Akishima, Tokyo, Japan). Scientific and common names of fish hosts follow FishBase (Froese and Pauly 2019). Terminology of microtriches follows Chervy (2009), that of scolex types Mackiewicz (1994; see his figs. 5.1-5.21).

Despite the considerable efforts of the present authors, it was not possible to collect representatives of all Nearctic genera. Therefore, material from museum collections was also studied. Voucher specimens of newly collected material and museum material are deposited in the Helminthological Collection of the Institute of Parasitology, Biology Centre of the Czech Academy of Sciences, České Budějovice, Czech Republic (IPCAS) and in the Smithsonian National Museum of Natural History, Washington, DC, USA (USNM; now hosting the previous U.S. National Parasite Collection formerly in Beltsville, Maryland - USNPC).

A list of specimens studied follows below. Species for which material was not fixed with hot fixative are marked with an asterisk (*). 
A

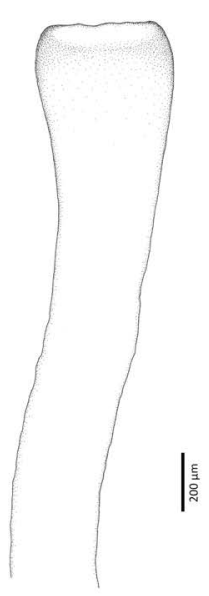

E

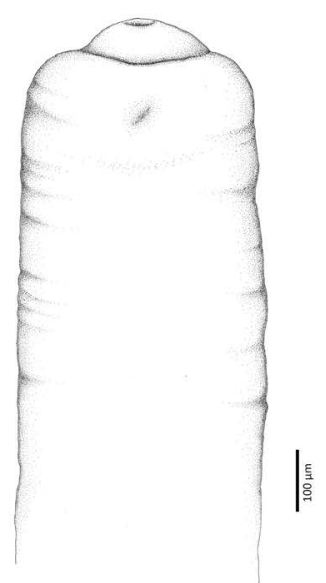

B

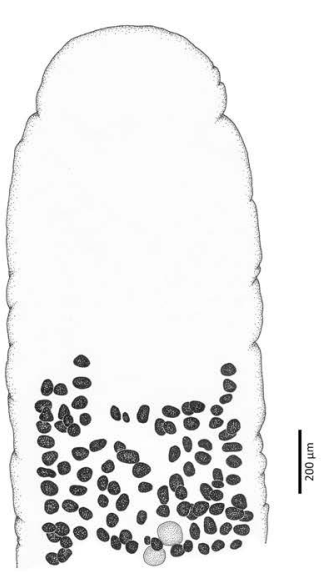

$\mathbf{F}$

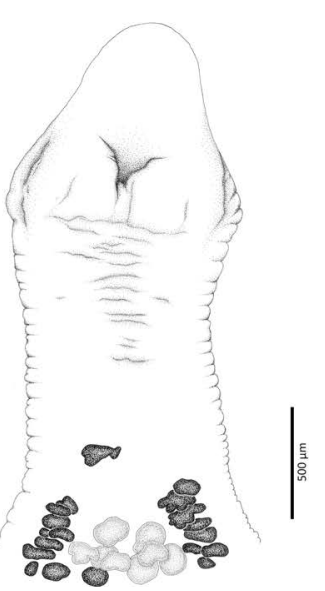

C
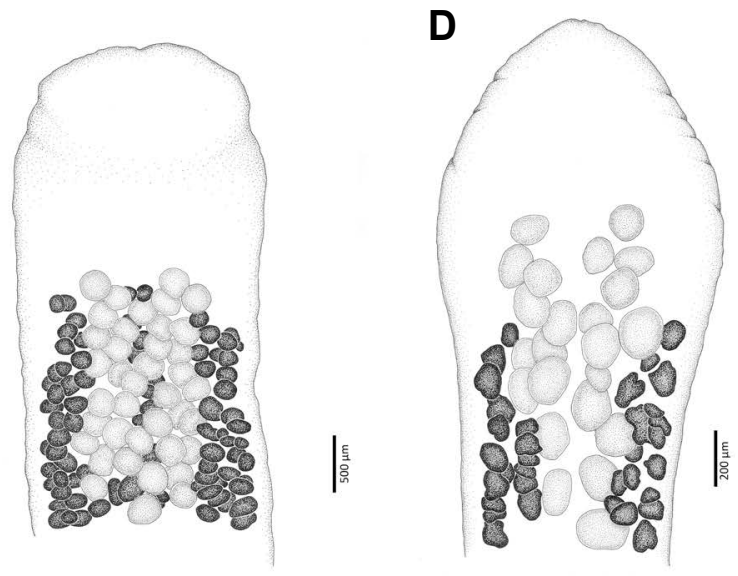

G

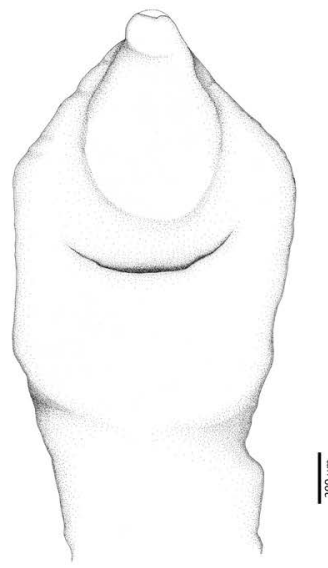

H

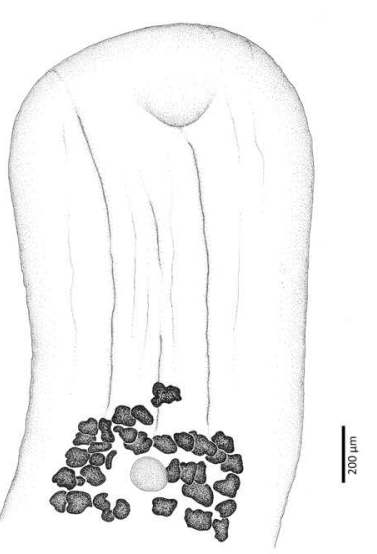

Fig. 2. Line drawings of the anterior parts of the Nearctic species of the Caryophyllidea with the anteriormost vitelline follicles (black) and testes (stippled). A - Calentinella etnieri Mackiewicz, 1974 ex Erimyzon claviformis (Girard); B - Caryophyllaeides fennica (Schneider, 1902) ex Acrocheilus alutaceus Agassiz et Pickering; C - Edlintonia ptychocheila Mackiewicz, 1970 ex Ptychocheilus oregonensis (Richardson); D - Hunterella nodulosa Mackiewicz et McCrae, 1962 ex Hypentelium nigricans (Le Sueur); E - Promonobothrium hunteri (Mackiewicz, 1963) ex Hypentelium nigricans; F - Pseudolytocestus differtus Hunter, 1929 ex Ictiobus bubalus (Rafinesque); G - Dieffluvium unipapillatum Williams, 1978 ex Moxostoma carinatum (Cope); H - Pliovitellaria wisconsinensis Fischthal, 1951 ex Notemigonus crysoleucas (Mitchill).

\section{Family Capingentidae Hunter, 1930}

1. Capingens singularis Hunter, 1927 from notchlip redhorse, Moxostoma collapsum (Cope)(host code US 274a/PBI [Planetary Biodiversity Inventory code of molecular voucher] 470), Congaree River at Columbia, South Carolina, USA.

2. Edlintonia ptychocheila Mackiewicz, 1970 from northern pikeminnow, Ptychocheilus oregonensis (Richardson), Bonner Co., Idaho, USA (IPCAS C-553/1); from peamouth, Mylocheilus caurinus (Richardson), Middle Fork Willamette River, Oregon, USA.

3. ${ }^{\star}$ Pseudolytocestus differtus Hunter, 1929 from smallmouth buffalo, Ictiobus bubalus (Rafinesque), Tallahatchie River, Mississippi, USA (holotype; USNM 1350001).

4. ${ }^{*}$ Spartoides wardi Hunter, 1929 from river carpsucker, Carpiodes carpio (Rafinesque), North Platte River, Nebraska, USA (IPCAS C-545/1).

\section{Family Caryophyllaeidae Leuckart, 1878}

5. ${ }^{\star}$ Archigetes iowensis Calentine, 1962 from red worm, Limnodrilus hoffmeisteri Claparède USA (IPCAS C-588).
6. Biacetabulum carpiodi Mackiewicz, 1969 from black buffalo, Ictiobus niger (Rafinesque) (US 244b/PBI 417), Chotard Lake, Vicksburg, Mississippi, USA.

7. Biacetabulum infrequens Hunter, 1927 from spotted sucker, Minytrema melanops (Rafinesque) (US 217b/PBI 415), Fish Lake near Pascagoula River at Benndale, Mississippi, USA.

8. Calentinella etnieri Mackiewicz, 1974 from western creek chubsucker, Erimyzon claviformis (Girard) (US 668), Sugar Creek, Cross Co., Arkansas, USA (IPCAS C-550/2).

9. ${ }^{\star D i e f f l u v i u m ~ u n i p a p i l l a t u m ~ W i l l i a m s, ~} 1978$ from river redhorse, Moxostoma carinatum (Cope), Cahaba River, Dallas Co., Alabama, USA (holotype; USNM 1369056).

10. Glaridacris catostomi Cooper, 1920 from white sucker, $\mathrm{Ca}$ tostomus commersonii, Assiniboine River, Manitoba, Canada (IPCAS C-5/1).

11. Hunterella nodulosa Mackiewicz et McCrae, 1962 from northern hogsucker, Hypentelium nigricans (Le Sueur), Waupaca River, Wisconsin, USA (IPCAS C-321); from Catostomus commersonii (DP 134), Duck Creek, Ashwaubenon and De Pere, Wisconsin, USA. 
A

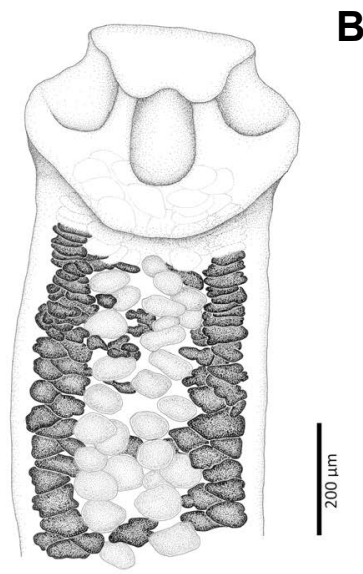

B

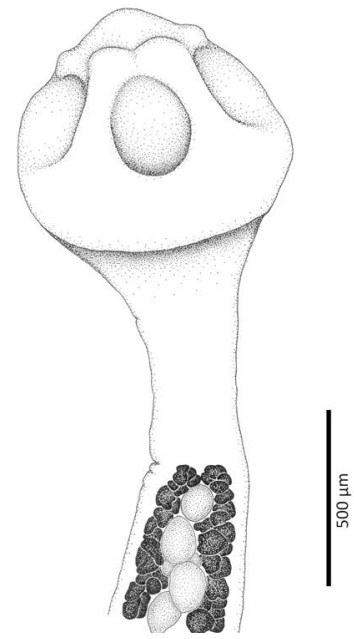

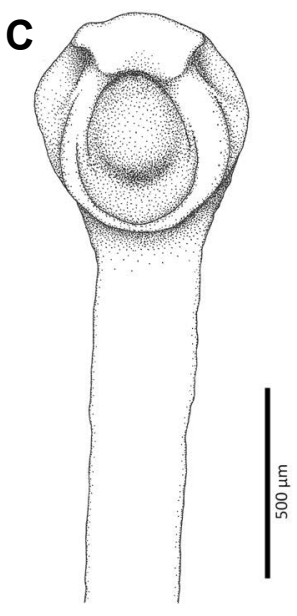

Fig. 3. Line drawings of the anterior parts of the Nearctic species of the Caryophyllidea with the anteriormost vitelline follicles (black) and testes (stippled). A - Archigetes iowensis Calentine, 1962 ex Limnodrilus hoffmeisteri (Claparède); B - Biacetabulum carpiodi Mackiewicz, 1969 ex Ictiobus niger (Rafinesque); C - Biacetabulum infrequens Hunter, 1927 ex Minytrema melanops (Rafinesque).

12. ${ }^{\star}$ Hypocaryophyllaeus gilae Fischthal, 1953 from Utah chub, Gila atraria (Girard), Emma Matilda River, Teton Co., Wyoming, USA (USNM 1347575).

13. Isoglaridacris wisconsinensis Williams, 1977 from shorthead redhorse, Moxostoma macrolepidotum (Le Sueur) (US 282a), Congaree River at Columbia, South Carolina, USA (PCAS C-652/1); from H. nigricans (DP 148/09b/PBI 413), Waupaca River, Wisconsin, USA.

14. Janiszewskella fortobothria Mackiewicz et Deutsch, 1976 from Carpiodes cyprinus (Le Sueur), Susquehanna River, Pennsylvania, USA (IPCAS C-529/1).

15. ${ }^{*}$ Penarchigetes oklensis Mackiewicz, 1969 from Ictiobus bubalus, North Eastern Outing Club Lake, Cherokee Co., Oklahoma, USA (IPCAS C-555/1) and from M. melanops, Oxbow Lake of Illinois River, Cherokee Co., Oklahoma, USA (holotype; USNM 1366840).

16. Pliovitellaria wisconsinensis Fischthal, 1951 from golden shin er, Notemigonus crysoleucas (Mitchill), Westhampton, Massachusetts, USA (IPCAS C-533/1); from northern redbelly dace, Chrosomus eos Cope, Bolger Bog, Upper Peninsula of Michigan, Michigan, USA.

17. Promonobothrium currani Oros, Brabec, Kuchta, Choudhury et Scholz, 2016 from I. niger (US 244c/PBI 460), Chotard Lake, Mississippi, USA (IPCAS C-696/2).

18. Promonobothrium hunteri (Mackiewicz, 1963) from H. nigricans, Grindstone Creek, New York, USA (IPCAS C-505/3).

19. Promonobothrium rogersi (Williams, 1980) from highfin carpsucker, Carpiodes velifer (Rafinesque) (US 216a/PBI 472), Pascagoula River, Mississippi, USA (IPCAS C-698).

20. Pseudoglaridacris confusa (Hunter, 1929) from Ictiobus cyprinellus (Valenciennes) (US 246a/PBI 461), Chotard Lake, Mississippi, USA (IPCAS C-363/3); from Ictiobus bubalus (US 245b/PBI 463), Chotard Lake, Mississippi, USA.

21. Pseudoglaridacris laruei (Lamont, 1921) from Moxostoma anisurum (Rafinesque) (DP 93/09a, 111/09a), Wolf River, Wisconsin, USA (IPCAS C-322/3); from Catostomus commersonii (US 66a), Lake Winnebago, Wisconsin, USA (IPCAS C-322/2)

22. Rowardleus pennensis Mackiewicz et Deutsch, 1976 from $C$. cyprinus (PBI 405), Lake Winnebago, Wisconsin, USA (IPCAS C-545).

\section{Family Lytocestidae Hunter, 1927}

23. Atractolytocestus huronensis Anthony, 1958 from common carp, Cyprinus carpio Linnaeus, Reelfoot Lake, Tennessee, USA (IPCAS C-472).

24. Caryophyllaeides fennica (Schneider, 1902) from chiselmouth, Acrocheilus alutaceus Agassiz et Pickering, Trout Creek, Oregon, USA (IPCAS C-2/20).

25. Khawia japonensis (Yamaguti, 1934) (syn. K. iowensis Calentine et Ulmer, 1961) from C. carpio, Mohawk River, Albany, New York, USA and Lake Texoma, Oklahoma, USA (IPCAS C-528).

No specimens of species of Bialovarium Fischthal, 1953 and Homeomorpha Dutton et Barger, 2014 were available. For characterisation and line drawings of their scoleces, the original descriptions were used. In addition, the two invasive species $A$. huronensis and $K$. japonensis, previously reported in detail (see Oros et al. 2010, 2015, Scholz et al. 2011), were also included in the present study.

\section{RESULTS}

Drawings of the scoleces (and the anterior extent of the testes and vitelline follicles in some of them) of 23 representative species of 20 genera are provided in Figs. 1-4. Scanning electron micrographs (SEM) of the scoleces of 14 species of 11 genera of caryophyllidean cestodes of all three families from North American catostomid and cyprinid fishes are presented in Figs. 5, 6. The following types of the scoleces can be recognised:

\section{Afossate}

1.1. bulboacuminate (Atractolytocestus - Fig. 1A);

1.2. cuneifimbriate (Khawia Hsü, 1935 - Fig. 1D);

1.3. cuneiform (Calentinella Mackiewicz, 1974, Caryophyllaeides Nybelin, 1922, Edlintonia Mackiewicz, 1970 Figs. 2A-C, 5A,C);

1.4. hastate (Pseudolytocestus Hunter, 1929 - Fig. 2F);

1.5. tholate (Hunterella Mackiewicz et McCrae, 1962 - Figs. 2D, 5B). 
A

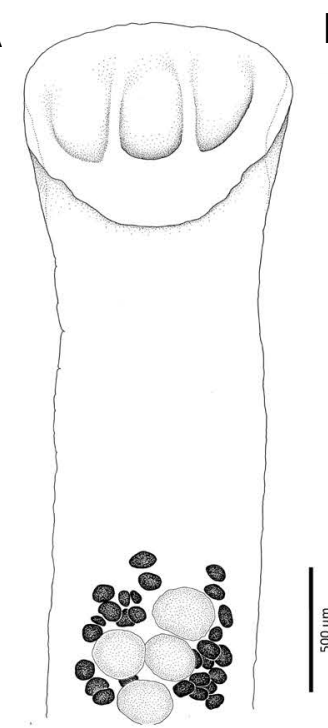

E

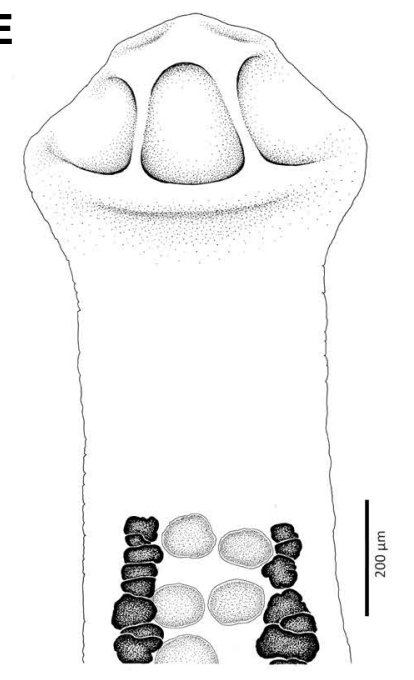

I

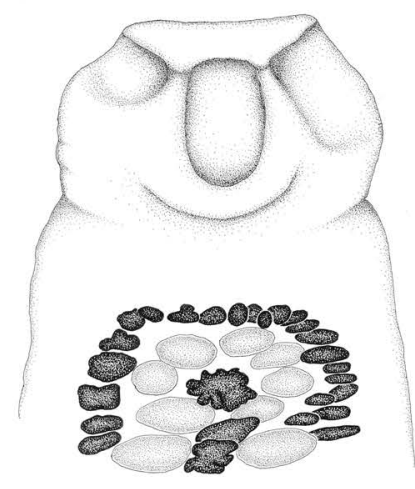

B

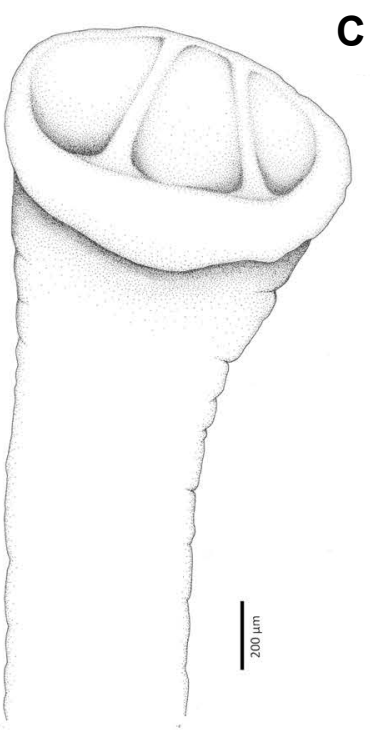

F

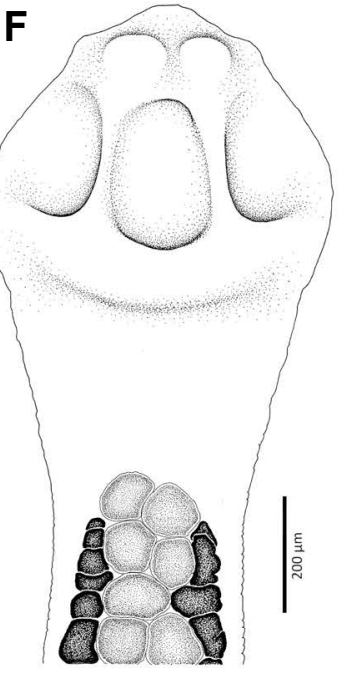

J

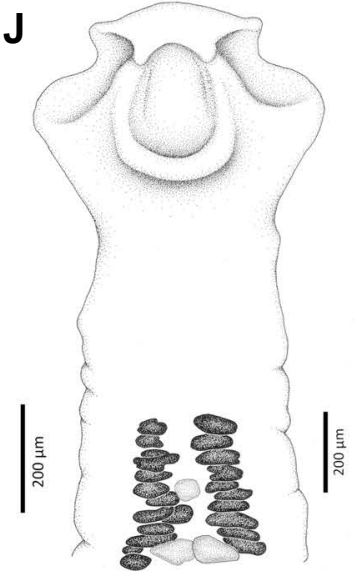

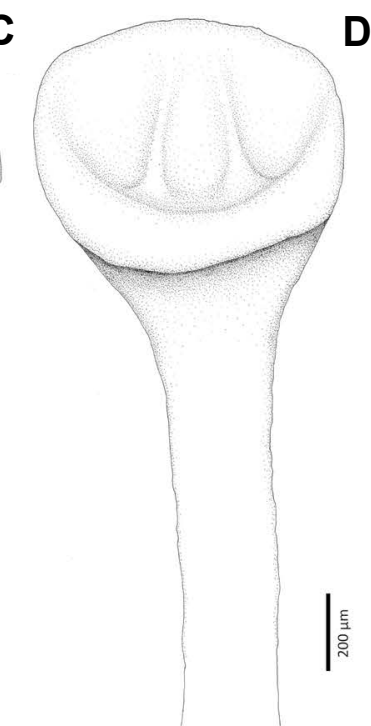

G

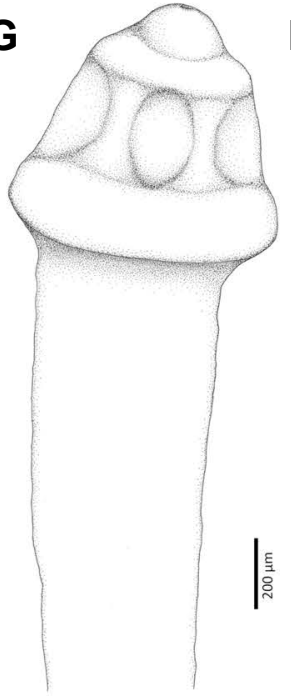

K

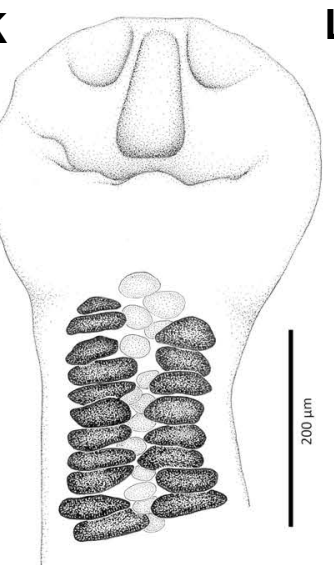

D

H
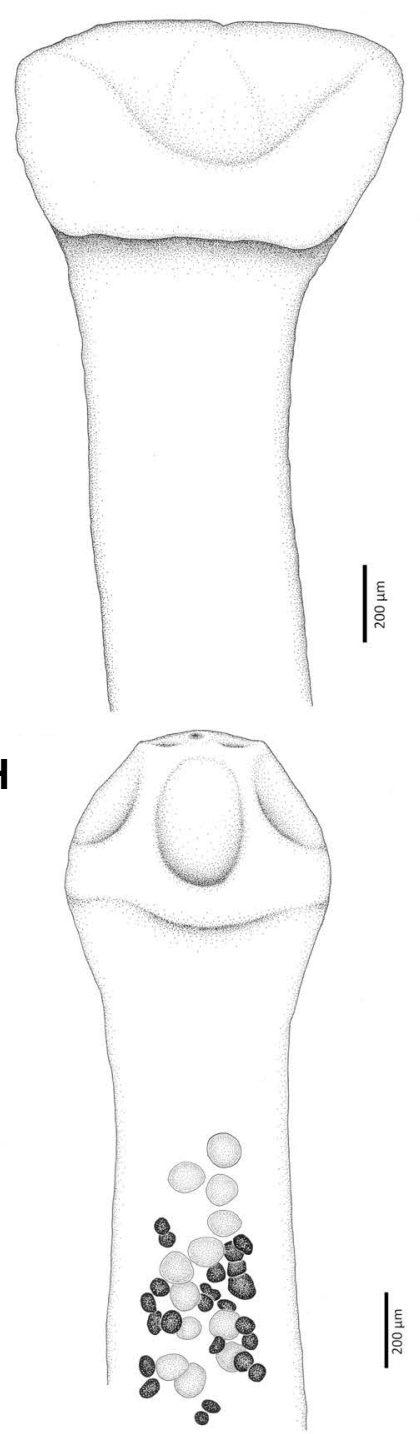

L

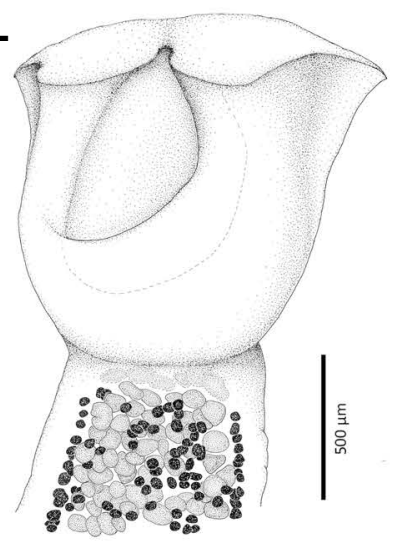

Fig. 4. Line drawings of the anterior parts of the Nearctic species of the Caryophyllidea with the anteriormost vitelline follicles (black) and testes (stippled). A - Glaridacris catosomi Cooper, 1920 ex Catostomus commersonii (Lacépède); B - Isoglaridacris wisconsinensis Williams, 1977 ex Moxostoma macrolepidotum (Le Sueur); C - Rowardleus pennensis Mackiewicz et Deutsch, 1976 ex Carpiodes cyprinus (Le Sueur); D - Spartoides wardi Hunter, 1929 ex Carpiodes carpio (Rafinesque); E - Pseudoglaridacris laruei (Lamont, 1921) ex Catostomus commersonii; F - Pseudoglaridacris confusa (Hunter, 1929) ex Ictiobus cyprinellus (Valenciennes); G - Promonobothrium currani Oros, Brabec, Kuchta, Choudhury et Scholz, 2016 ex Ictiobus niger (Rafinesque); H - Promonobothrium rogersi (Williams, 1980) ex Carpiodes velifer (Rafinesque); I - Hypocaryophyllaeus gilae Fischthal, 1953 ex Gila atraria (Girard); J-Janiszewskella fortobothria Mackiewicz et Deutsch, 1976 ex Carpiodes cyprinus; K-Penarchigetes oklensis Mackiewicz, 1969 ex Minytrema melanops (Rafinesque); L - Capingens singularis Hunter, 1927 ex Moxostoma collapsum (Cope). 


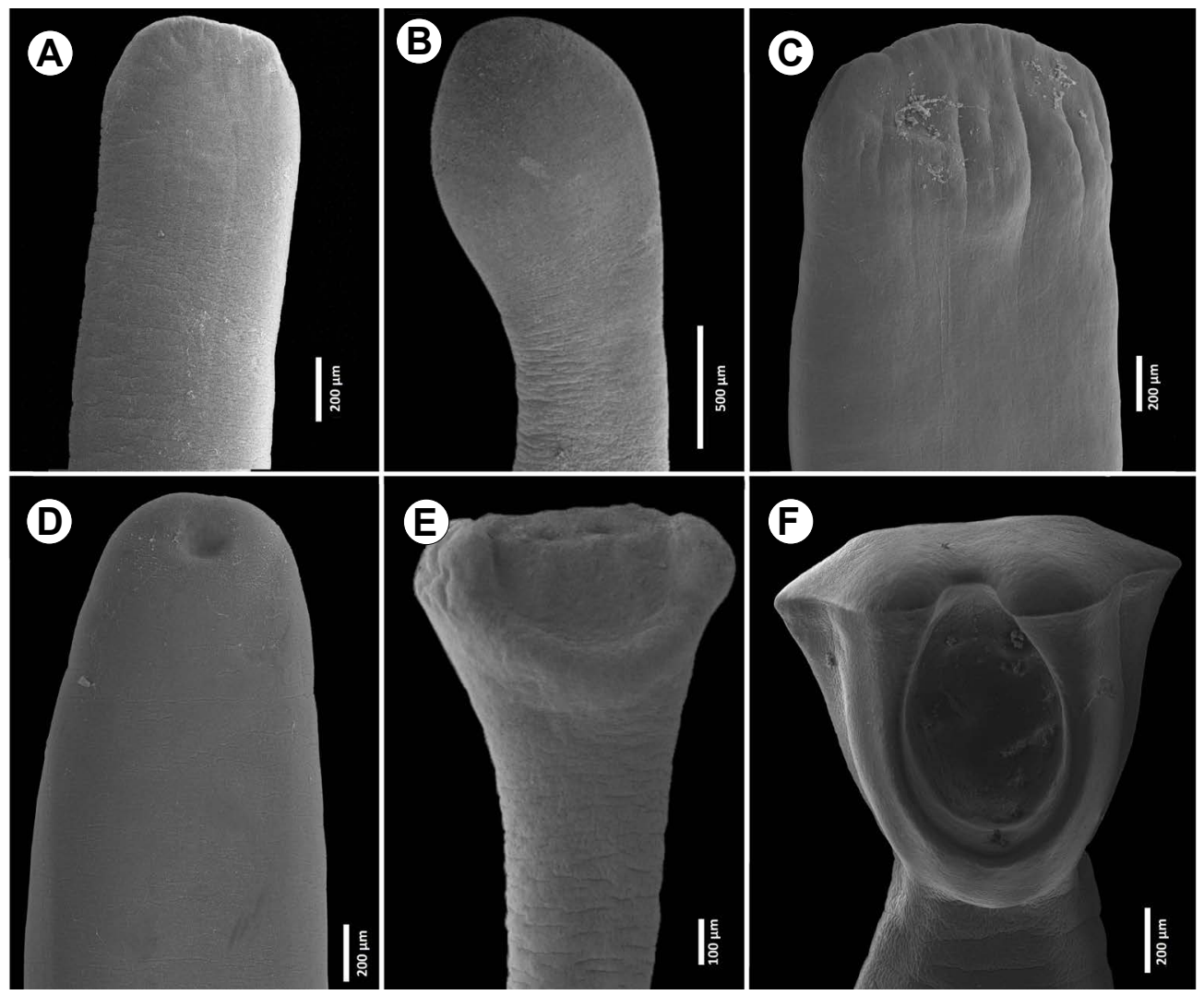

Fig. 5. Scanning electron photomicrographs of the scoleces of the Nearctic species of Caryophyllidea. A - Caryophyllaeides fennica (Schneider, 1902) ex Acrocheilus alutaceus Agassiz et Pickering; B - Hunterella nodulosa Mackiewicz et McCrae, 1962 ex Catostomus commersonii (Lacépède); C - Edlintonia ptychocheila Mackiewicz, 1970 ex Mylocheilus caurinus (Richardson); D - Pliovitellaria wisconsinensis Fischthal, 1951 ex Chrosomus eos Cope; E - Spartoides wardi Hunter, 1929 ex Carpiodes carpio (Rafinesque); F - Capingens singularis Hunter, 1927 ex Moxostoma collapsum (Cope).

\section{Fossate}

2.1. biacetabulate (Biacetabulum - Figs. 3C, 6D);

2.2. bothrioloculodiscate (Archigetes Leuckart, 1878, Biacetabulum, Janiszewskella Mackiewicz et Deutsch, 1976, Penarchigetes Mackiewicz, 1969, Pseudoglaridacris Oros, Uhrovič et Scholz, 2018 - Figs. 3A, 4E,F,J,K, 6F,G);

2.3. bulboloculate (Biacetabulum, Janiszewskella - Figs. 3B, $4 \mathrm{~J}, 6 \mathrm{H})$;

2.4. cuneiformoloculate (Glaridacris Cooper, 1920 - Figs. $4 \mathrm{~A}, 6 \mathrm{C})$;

2.5.cuneiloculate (Homeomorpha, Hypocaryophyllaeus Hunter, 1927, Isoglaridacris, Rowardleus Mackiewicz et Deutsch, 1976, Spartoides Hunter, 1929 - Figs. 1C, $4 \mathrm{~B}-\mathrm{D}, \mathrm{I}, 5 \mathrm{E}, 6 \mathrm{~B})$;

2.6. digitiform-loculate (Promonobothrium - Figs. 2E, 6A);

2.7. fixomegabothriate (Capingens Hunter, 1927- Figs. 4L, $5 \mathrm{~F})$;

2.8. loculotholate (Bialovarium, Pliovitellaria Fischthal, 1951 - Figs. 1B, 2H, 5D);

2.9. loculotruncate (Dieffluvium - Fig. 2G, Promonobothrium - Fig. $4 \mathrm{H})$;

2.10. loculomonobothriate (Promonobothrium - see fig. 1B in Oros et al. 2016)

2.11. monobothriate (Promonobothrium - Fig. 2E, 6A)

\section{Characterisation of the scoleces of caryophyllidean genera from the Nearctic Region}

\section{Family Capingentidae Hunter, 1930 (4 genera)}

Capingens Hunter, 1927

Figs. 4L, 5F

Scolex fixomegabothriate - massive and robust, about $1 / 5$ to $1 / 4$ of total body length, with large, well-developed ventral and dorsal bothria; first testes begin at base of scolex.

Edlintonia Mackiewicz, 1970

Figs. 2C, 5C

Scolex cuneiform - simple, aloculate, chisel-shaped, sagittate in lateral view; neck absent; first vitelline follicles begin posterior to first testes.

Pseudolytocestus Hunter, 1929

Fig. 2F

Scolex weakly hastate, aloculate - slightly sagittate, partially narrowed in neck; first testes begin posterior to first vitelline follicles.

Spartoides Hunter, 1929

Figs. 4D, 5E

Scolex cuneiloculate - distinct, with three pairs of shallow loculi, sagittate in lateral view; neck long, narrower than scolex; first testes begin posterior to first vitelline follicles. 

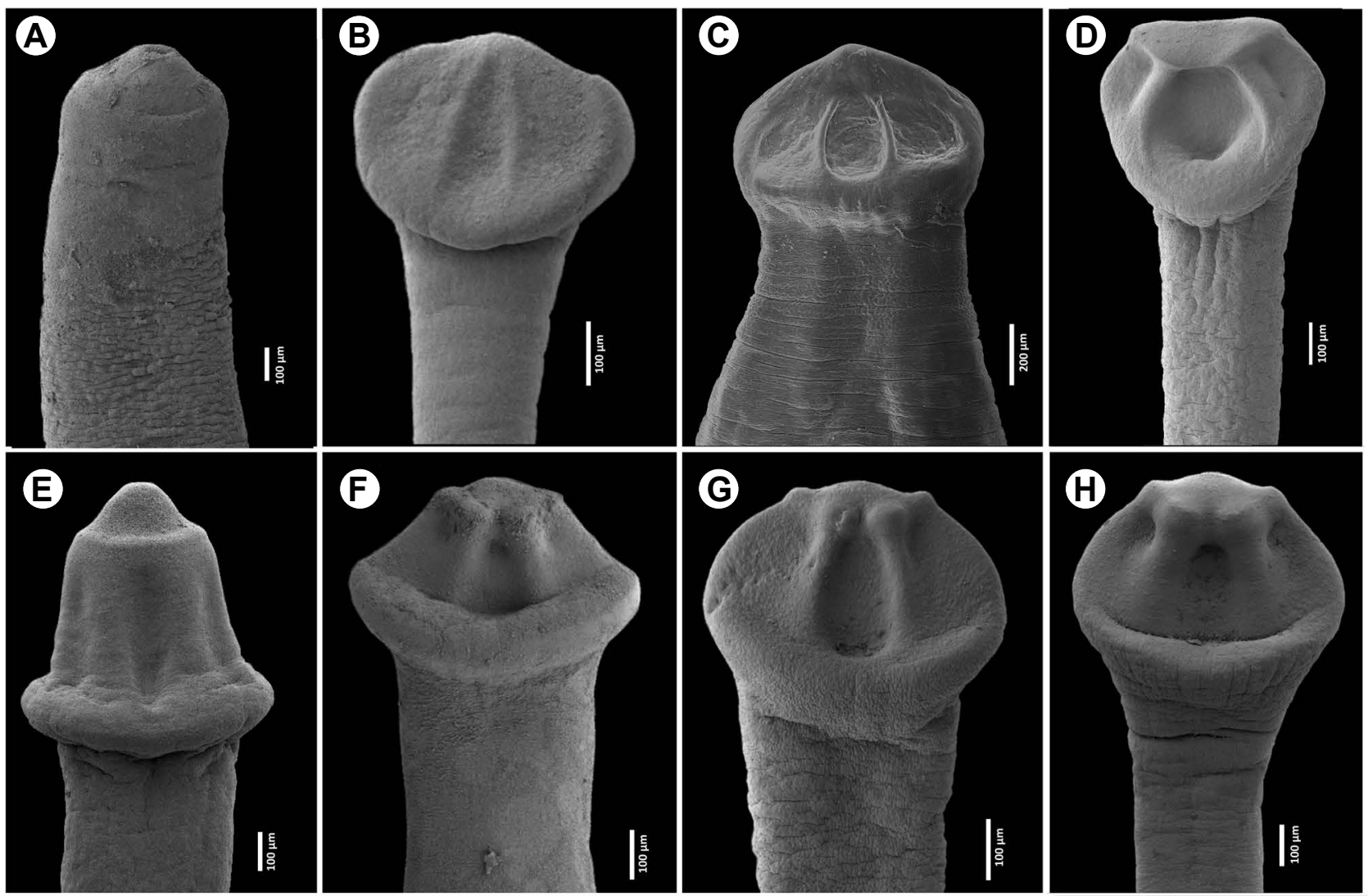

Fig. 6. Scanning electron photomicrographs of the scoleces of Nearctic species of Caryophyllidea. A - Promonobothrium hunteri (Mackiewicz, 1963) ex Hypentelium nigricans (Le Sueur); B - Isoglaridacris wisconsinensis Williams, 1977 ex Moxostoma anisurum (Rafinesque); C - Glaridacris catostomi Cooper, 1920 ex Catostomus commersonii (Lacépède); D - Biacetabulum infrequens Hunter, 1927 ex Minytrema melanops (Rafinesque); E - Promonobothrium currani Oros, Brabec, Kuchta, Choudhury et Scholz, 2016 ex Ictiobus niger (Rafinesque); F - Pseudoglaridacris laruei (Lamont, 1921) ex Moxostoma anisurum; $\mathbf{G}$ - Pseudoglaridacris confusa (Hunter, 1929) ex Ictiobus bubalus (Rafinesque); H - Biacetabulum carpiodi Mackiewicz, 1969 ex Ictiobus niger.

\section{Family Caryophyllaeidae Leuckart, 1878 (16 genera)}

Archigetes Leuckart, 1878

Fig. 3A

Scolex bothrioloculodiscate - spherical with two pairs of deep loculi and a pair of deep central depressions, terminal disc present; neck absent; first testes begin at base of scolex, first vitelline follicles begin posterior to first testes.

Biacetabulum Hunter, 1927

Figs. 3B,C, 6D,H

Scolex bulboloculate, bothrioloculodiscate, biacetabulate - relatively large scolex slightly wider than body, with or without one pair of deep central acetabulum-like structure and two pairs of less pronounced lateral loculi or just one pair of weak loculi present on scolex; variability of shape and type of scoleces very broad.

\section{Bialovarium Fischthal, 1953}

Fig. 1B

Scolex loculotholate - poorly defined, blunt, bearing a pair of shallow loculi, dorsoventrally compressed; neck absent; first testes begin posterior to first vitelline follicles.

\section{Calentinella Mackiewicz, 1974}

Fig. 2A

Scolex cuneiform - blunt-ended, slightly dorsoventrally compressed, wider than neck; neck present, long; first testes begin posterior to first vitelline follicles.
Dieffluvium Williams, 1978

Fig. 2G

Scolex loculotruncate - expanded posteriorly within the pair of loculi in expanded portion; neck present, long; first testes begin posterior to first vitelline follicles.

\section{Glaridacris Cooper, 1920}

Figs. 4A, 6C

Scolex cuneiformoloculate - wedge-shaped, with three shallow loculi on dorsal and ventral side of scolex; neck present, short; first testes begin posterior to first vitelline follicles.

\section{Homeomorpha Dutton et Barger, 2014}

Fig. 1C

Scolex cuneiloculate - shallowly obovoid, with three pairs of shallow loculi; neck present, long; first testes begin posterior to first vitelline follicles.

\section{Hunterella Mackiewicz et McCrae, 1962 Figs. 2D, 5B}

Scolex tholate - simple, non-specialised with round to conical shape; neck absent; first testes begin immediately posterior to scolex, vitelline follicles begin posterior to first testes.

\section{Hypocaryophyllaeus Hunter, 1927}

Fig. 4I

Scolex cuneiloculate - indistinctly defined from neck, bearing a flattened terminal disc and three pairs of distinct 
loculi; neck absent; first vitelline follicles begin anterior to first testes.

\section{Isoglaridacris Mackiewicz, 1965}

Figs. 4B, 6B

Scolex cuneiloculate - fan-shaped, with three pairs of shallow loculi; neck present; first testes begin posterior to first vitelline follicles.

Janiszewskella Mackiewicz et Deutsch, 1976

Fig. 4J

Scolex bothrioloculodiscate - slightly spherical with two pairs of deep loculi and pair of deep central bothria; neck short; first testes begin posterior to first vitelline follicles.

\section{Penarchigetes Mackiewicz, 1969}

Fig. 4K

Scolex bothrioloculodiscate - slightly cylindrical or conical, wider than the body, with one pair of median loculus and two pairs of lateral loculi, apical disc small; neck indistinct or distinct; vitelline follicles begin slightly anterior or posterior to first testes, to posterior end of scolex or to neck region only.

\section{Pliovitellaria Fischthal, 1951}

Figs. 2H, 5D

Scolex loculotholate - rounded, dorsoventralilly compressed, with one central shallow depression, wider than the body; neck absent; first testes begin posterior to first vitelline follicles.

\section{Promonobothrium Mackiewicz, 1968 Figs. 4G,H, 6E}

Scolex digitiform, digitiform-loculate or loculotruncate - with a terminal introvert on apex and one or three pair(s) of shallow dorsoventral depressions.; neck short to moderately long; variability of shape of scoleces and arrangement of vitelline follicles and testes very broad.

\section{Pseudoglaridacris Oros, Uhrovič et Scholz, 2018}

Figs. 4E,F, 6F,G

Scolex bothrioloculodiscate - forming definite terminal (apical) disc and three pairs of loculi; relatively short neck; variability of anterior position of vitelline follicles and testes broad.

\section{Rowardleus Mackiewicz et Deutsch, 1976}

Fig. 4C

Scolex cuneiloculate - round to ellipsoidal, with three pairs of featureless loculi in one pair shallow depression; neck region long; first testes begin posterior to first vitelline follicles.

\section{Family Lytocestidae Hunter, 1927}

(3 genera)

Atractolytocestus Anthony, 1958

Fig. 1A

Scolex bulboacuminate - arrowhead-shaped (tapered) or bluntly ended, with smooth anterior margin, scolex wider than distinct neck; first testes begin posterior to vitelline follicles.

\section{Caryophyllaeides Nybelin, 1922}

Figs. 2B, 5A

Scolex cuneiform - blunt-ended, slightly dorsoventral compressed, aloculate; neck absent; first testes begin posterior to first vitelline follicles.

\section{Khawia Hsü, 1935}

Fig. 1D

Scolex cuneifimbriate - lacking apical structures and lateral loculi, with frilled or deeply folded anterior margin, scolex wider than neck region; first testes and first vitelline follicles start at approximately same level, or first testes begin posterior to first vitelline follicles.

\section{DISCUSSION}

The present study has confirmed high diversity of scolex types in the Nearctic species of caryophyllidean cestodes. In contrast, species from other zoogeographical regions are rather uniform in scolex morphology (see Oros et al. 2010 for Palaearctic species, Ash et al. 2011a,b for Indomalayan taxa). As many as 16 types of scoleces can be recognised among Nearctic taxa, but intrageneric variation may differ considerably between individual genera. Species of most genera have rather uniform shape (31 species studied), but the genus Promonobothrium as recently re-defined by Oros et al. (2016) includes taxa with conspicuously different scolex types, i.e. monobothriate, loculotruncate, biacetabulate and digitiform-loculate (Figs. 2E, 4G,H, 6E). As new data from further collections and molecular analysis become available, changes will be necessary for terminology of scolex types.

The scolex of caryophyllidean tapeworms is highly mobile and variable, with no single type being characteristic of this group (Mackiewicz 1970, 1972, 1994). Mackiewicz (2003) distinguished two basic scolex types among caryophyllideans of the world and indicated that 24 genera (59\%) of all caryophyllideans recognised at that time possess afossate scoleces and 17 genera (41\%) possess fossate scoleces. As obvious from the present data, the number of afossate genera among only Nearctic caryophyllideans is much lower (only 7 genera out of 23 , i.e. $30 \%$ ).

The scolex has been suggested as a useful structure for generic and/or species identification (Kulakovskaya 1961, Chubb et al. 1987, Dubinina 1987, Scholz 1989, Protasova 1990, Oros et al. 2010), but the use of scolex morphology can be limited in some taxa. A detailed study of two species of the Palaearctic genus Caryophyllaeus, C. brachycollis Janiszewska, 1953 and C. laticeps (Pallas, 1781), has revealed high plasticity in their scolex morphology (Barčák et al. 2014, Hanzelová et al. 2015). This intraspecific, possibly host-related morphological variability represents a serious obstacle for reliable identification of species of this genus (Barčák et al. 2017).

The present study of Nearctic species has shown that form and shape of the scolex may help in differentiating most Nearctic genera, especially those with a low number of taxa that have a unique form of scolex (e.g., Capingens, Hunterella, Pliovitellaria and Pseudolytocestus). However, it should be mentioned that different methods of fixation may greatly influence scolex morphology (Oros et al. 2010). Live material should also be studied to observe the natural extension and variation of the scolex during normal contractions (Mackiewicz 1972). For example, species of Pseudoglaridacris are characterised by a bothrioloculodiscate scolex, i.e. the scolex forming a definite terminal (apical) disc and three pairs of loculi. However, the normal 
flat terminal disc may naturally push forward into a dome configuration and the rest of the scolex may have knobs formed by the expanded muscles that form the disc (Oros et al. 2018). This abnormal extentsion can be readily seen, for example, in species of Pseudoglaridacris (Fig. 4E,F) and Biacetabulum (Fig. 3B,C) of the present study.

Examination of the holotype of Dieffluvium unipapillatum (USNM 1369056) and two other gravid individuals determined to be that species indicates that the scolex of the holotype has been compressed and misshapen. There is no papilla-like structure as described and incorrectly illustrated by Williams (1978). Based on non-compression, we interpret the scolex to be most similar to the loculotruncate type. As a result the previous designation of loculopapillate is considered inappropriate and inaccurate and has been based on an abnormal, compressed scolex. As a consequence of this mistake in terminology, the term and figures described of Williams (1978: figs 1 and 5) as loculopapillate and subsequently utilised by Mackiewicz (1994) and Hoffman (1999) should no longer be used. A similar observation has been made independently by Herzog and Barger (2019).

A robust phylogenetic hypothesis of relationships of caryophyllidean cestodes is not yet available (see Scholz and Oros 2017 for preliminary data on interrelationships of these cestodes). Brabec et al. (2012) included in their phylogenetic analyses only three Nearctic taxa, namely Glaridacris catostomi, Hunterella nodulosa and Promonobothrium hunteri (as Monobothrium hunteri Mackiewicz, 1963), all of the family Caryophyllaeidae. Scholz et al. (2015) and Oros et al. (2016) enlarged the number of species of Promonobothrium, but no representatives of other Nearctic genera were analysed. Therefore, it is not possible to reliably assess the homology of individual types of scoleces of Nearctic caryophyllideans, which possess structures absent in species from other zoogeographical regions (Mackiewicz 1972, Oros et al. 2010). Preliminary molecular phylogenetic data generated by A. Waeschenbach (see Scholz and Oros 2017) indicate that Nearctic taxa form a monophyletic, more recently diverging group distant from the remaining caryophyllideans from other zoogeographical regions.

The presence of the same scolex type in genera of different families (cuneiform in the capingentid Edlintonia, caryophyllaeid Calentinella and lytocestid Caryophyllaeides, and cuneiloculate in the capingentid Spartoides and caryophyllaeids Hypocaryophyllaeus and Rowardleus) indicates that scolex morphology does not necessarily reflect the evolutionary history and relatedness of these taxa. Indeed, the current system of families is undoubtedly artificial as evidenced by several studies (Oros et al. 2008, 2016, Brabec et al. 2012, Scholz and Oros 2017). Similarly as in some other cestode groups (e.g., Scholz et al. 2013), the scolex morphology can be a result of adaptation to a particular host physiology, intestinal region or intestinal surface architecture rather than it witnesses co-evolutionary history of taxa with a similar type of scolex. Interestingly, North American caryophyllideans from cyprinids, i.e. species of Atractolytocestus, Caryophyllaeides, Edlintonia, Khawia and Pliovitellaria, have simpler scoleces compared to those of caryophyllideans from catostomids.

Scoleces of some species of Biacetabulum invariably possess a large, deep central structure on the dorsal and ventral sides with shallower, lateral loculi, but the shape and depth of loculi and central structure may differ between species and individual specimens (Figs. 3B,C, 5D,H). According to Hunter (1930), McCrae (1962), Mackiewicz (1972, 1994), Williams (1977), Mackiewicz et al. (1972), the central structure represents a pair of true acetabula that function like suckers, i.e. acetabulate attachment organs (small suckers) of more recently diverged 'tetraphyllidean-like' cestodes of Olson et al. (2001) and Caira and Jensen (2017) (see Mackiewicz et al. 1972: fig. 18 for photo of in situ attachment of the "acetabulum"). However, the anatomy of this pair of large structures, which appears to be well demarcated with a basement membrane only in its posterior part, does not seem to be homologous with acetabulate structures (four small suckers) of oncoproteocephalideans, cyclophyllideans and other tapeworms (see Olson et al. 2001, Caira et al. 2014, Caira and Jensen 2017). Most likely, the 'acetabulum' in species of Biacetabulum and Promonobothrium represents an example of convergent evolution of similar but larger pair of sucker-like attachment (acetabulate) structures in cestodes.

In addition to documenting extraordinary shape diversity of the scoleces of North American taxa using light and scanning electron microscopy, the present study has also revealed that the diversity of this cestode group is not fully known. Some of the specimens studied may represent new, undescribed taxa whose formal descriptions are pending. For a more conclusive study, examination of missing taxa, in particular those from endemic catostomids and cyprinids in the western part of North America, is also highly needed to generate a robust phylogenetic hypothesis of the group. Such new data would enable us to test homology of scolex forms in North American caryophyllideans and their importance for classification of these cestodes.

Acknowledgements. We thank Eric Hoberg and Pat Pilitt, both from the former U.S. National Parasite Collection (USNPC) in Beltsville, MD, USA, and Eileen Harris, Natural History Museum, London, UK, for enabling one of the present authors (T.S.) to study type and voucher specimens of North American caryophyllideans. Thanks are also due to Anirban Ash, India, who helped with examination of caryophyllideans in USNPC in 2008, Roman Kuchta, Czech Republic, Megan Bean, Texas, Isaure de Buron, South Carolina, Steve Curran, Eric Pulis and Robin M. Overstreet, Mississippi, for help with collecting fish cestodes in the United States. MO and AC thank Patrick Nelson and Doug Watkinson for help with sampling in Manitoba, Canada, and Ron Bruch, Ryan Koenigs and Ryan Zernzach for help with sampling in Wisconsin, USA. This study was partly supported by the Grant Agency VEGA (No. 2/0159/16), Ministry of Education, Sports and Youth (project No. LTAUSA18), Institute of Parasitology (RVO: 60077344) and European Commission, SYNTHESYS Project GB-TAF-1135 to T.S. Stay of M.O. and T.S. in North America in 2013 and 2017, respectively, was enabled by the Fulbright Commission. AC thanks St. Norbert College Faculty Development grants for support. 


\section{REFERENCES}

Ash A., Scholz T., Oros M., Kar P.K. 2011a: Tapeworms (Cestoda: Caryophyllidea), parasites of Clarias batrachus (Pisces: Siluriformes) in the Indomalayan Region. J. Parasitol. 97: 435-459.

Ash A., Scholz T., Oros M., Levron C., Kar P.K. 2011b: Cestodes (Caryophyllidea) of the stinging catfish Heteropneustes fossilis (Siluriformes: Heteropneustidae) from Asia. J. Parasitol. 97: 899-907.

Barčák D., Oros M., Hanzelová V., Scholz T. 2014: Phenotypic plasticity in Caryophyllaeus brachycollis Janiszewska, 1953 (Cestoda: Caryophyllidea): does fish host play a role? Syst. Parasitol. 88: 153-166.

BarČák D., Oros M., Hanzelová, V., Scholz T. 2017: A synoptic review of Caryophyllaeus Gmelin, 1790 (Cestoda: Caryophyllidea), parasites of cyprinid fishes. Folia Parasitol. 63: 027.

Brabec J., Scholz T., Králová-Hromadová I., BaszalovICSOvÁ E., OLSON P.D. 2012: Substitution saturation and nuclear paralogs of commonly employed phylogenetic markers in the Caryophyllidea, an unusual group of non-segmented tapeworms (Platyhelminthes). Int. J. Parasitol. 42: 259-267.

Caira J.N., Jensen K. (Eds) 2017: Planetary Biodiversity Inventory (2008-2017): Tapeworms from Vertebrate Bowels of the Earth. Natural History Museum, The University of Kansas, Natural History Museum, Lawrence, USA, 463 pp.

Caira J.N., Jensen K., Waeschenbach A., Olson P.D., LitTLEWOOD D.T.J. 2014: Orders out of chaos - molecular phylogenetics reveals the complexity of shark and stingray tapeworm relationships. Int. J. Parasitol. 44: 55-73.

Chervy L. 2009: Unified terminology for cestode microtriches: a proposal from the International Workshops on Cestode Systematics in 2002-2008. Folia Parasitol. 56: 199-230.

Chubb J.C., Pool D.W., Veltkamp C.J. 1987: A key to the species of cestodes (tapeworms) parasitic in British and Irish fresh-water fishes. J. Fish Biol. 31: 517-543.

Dubinina M.N. 1987. [Class tapeworms - Cestoda Rudolphi, 1808.] In: O.N. Bauer (Ed.), Key to the Parasites of Freshwater Fishes of the USSR.] Nauka, Leningrad, pp. 5-76. (In Russian.)

Dutton H.R., Barger M.A. 2014: A new genus and species of caryophyllaeid (Cestoda: Caryophyllidea) from spotted suckers, Minytrema melanops (Catostomidae), in the Big Thicket National Preserve, Texas, U.S.A. Comp. Parasitol. 81: 23-26.

Fischthal J.H. 1954: Bialovarium nocomis Fischthal, 1953 (Cestoda: Caryophyllaeidae) from hornyhead chub, Nocomis biguttatus (Kirtland). Proc. Helminthol. Soc. Wash. 21: 117-120.

Froese R., Pauly D. (Eds) 2019. Fishbase. World Wide Web electronic publication. http://www.fishbase.org.

Hanzelová V., Oros M., BarČák D., Miklisová D., Kirin D. SCHOLZ T. 2015: Morphological polymorphism in tapeworms: redescription of Caryophyllaeus laticeps (Cestoda: Caryophyllidea) and characterization of its morphotypes from different fish hosts. Syst. Parasitol. 90: 177-190.

HaYUnGa E.G., 1979: Observations on the intestinal pathology caused by three caryophyllid tapeworms of the white sucker $\mathrm{Ca}$ tostomus commersoni Lacépède. J. Fish Dis. 2: 239-248.

Hayunga E.G., MaCKIEWICZ J.S. 1988: Comparative histology of the scolex and neck region of Glaridacris laruei (Lamont, 1921) Hunter, 1927 and Glaridacris catostomi Cooper, 1920 (Cestoidea: Caryophyllidea). Can. J. Zool. 66: 790-803.

Herzog T.L., Barger M.A. 2019: A new species of Dieffluvium (Cestoda: Caryophyllidea) from smallmouth buffalo (Ictiobus bubalus) from the Big Thicket National Preserve, Texas, U.S.A Comp. Parasitol. 86: 73-78.

Hoffman G.L. 1999: Parasites of North American Freshwater Fishes. Second Edition. Cornell University Press, Ithaca, New York, $539 \mathrm{pp}$.
Hunter G.W. 1930: Studies on the Caryophyllaeidae of North America. Illinois Biological Monographs 11 (1927), University of Illinois, Illinois, $186 \mathrm{pp}$.

Khalil L.F., Jones A., Bray R.A. (Eds) 1994: Keys to the Cestode Parasites of Vertebrates. CAB International, Wallingford, $752 \mathrm{pp}$.

KulakovsKaya O.P. 1961: [Materials on the fauna of Caryophyllaeidae (Cestoda, Pseudophyllidea) of the Soviet Union.] Parazitol. Sbornik 20: 339-355. (In Russian.)

MACKIEWICZ J.S. 1970: Synopsis of caryophyllidean (Cestoda) morphology. J. Parasitol. 56 (4) sec. II: 220-221 + 1 Plate.

MACKIEWICZ J.S. 1972: Caryophyllidea (Cestoidea): a review. Exp. Parasitol. 34: 417-512.

MACKIEwiCZ J.S. 1994: Order Caryophyllidea van Beneden in Carus, 1863. In: L.F. Khalil, A. Jones and R.A. Bray (Eds), Keys to the Cestode Parasites of Vertebrates. CAB International, Wallingford, pp. 21-43.

MACKIEWICZ J.S. 2003: Caryophyllidea (Cestoidea): molecules, morphology and evolution. Acta Parasitol. 48: 143-154.

Mackiewicz J.S., Cosgrove G.E., Gude W.D. 1972: Relationship of pathology to scolex morphology among caryophyllid cestodes. Z. Parasitenkd. 39: 233-246.

Mackiewicz J.S., McCrae R.C. 1965: Biacetabulum biloculoides n. sp. (Cestoidea: Caryophyllaeidae) from Catostomus commersoni (Lacépède) in North America. Proc. Helminthol. Soc. Wash. 32: 225-228.

MCCrae R.C. 1962: Biacetabulum macrocephalum sp. n. (Cestoda: Caryophyllaeidae) from the white sucker Catostomus commersoni (Lacépède) in northern Colorado. J. Parasitol. 48: 807-811.

Olson P.D., Littlewood D.TJ., Bray R.A. Mariaux J. 2001: Interrelationships and evolution of the ta,peworms (Platyhelminthes: Cestoda). Mol. Phyl. Evol. 19: 443-467.

Oros M., Barčák D., Bazsalovicsová E., Hanzelová V. 2015: Asian fish tapeworm, Khawia japonensis (Yamaguti, 1934), has expanded its European invasive range. Parasitol. Res. 114: 2035-2039.

Oros M., Brabec J., Kuchta R., Choudhury A., Scholz T. 2016: A synoptic review of Promonobothrium Mackiewicz, 1968 (Cestoda: Caryophyllidea), parasites of suckers (Catostomidae) in North America, with description of two new species. Folia Parasitol. 63: 008.

Oros M., Scholz T., Hanzelová V., Mackiewicz J.S. 2010: Scolex morphology of monozoic cestodes (Caryophyllidea) from the Palaeartctic Region: a useful tool for species identification. Folia Parasitol. 57: 37-46.

Oros M., Uhrovič D., Scholz T. 2018: A new classification of Glaridacris Cooper, 1920 (Cestoda: Caryophyllidea), parasites of suckers (Catostomidae) in North America, including erection of Pseudoglaridacris n. gen. J. Parasitol. 104: 60-69.

Protasova E.P., Kuperman B.I., Roitman V.A., Poddubnaya L.G. 1990: [Caryophyllid Tapeworms of the Fauna of USSR.] Nauka, Moscow, 237 pp. (In Russian.)

SCHMidT G.D. 1986: CRC Handbook of Tapeworm Identification. CRC Press, Inc., Boca Raton, 675 pp.

Scholz T. 1989: Amphilinida and Cestoda, parasites of fish in Czechoslovakia. Acta Sci. Nat. Acad. Sci. Bohemoslov. Brno 23: $1-56$.

Scholz T., Brabec J., Králová-Hromadová I., Oros M., Bazsalovicsová E., Ermolenko A., Hanzelová V. 2011: Revision of Khawia spp. (Cestoda: Caryophyllidea), parasites of cyprinid fish, including a key to their identification and molecular phylogeny. Folia Parasitol. 58: 197-223.

Scholz T., De Chambrier A., Kuchta R., Littlewood D.T.J., WAESCHENBACH A. 2013: Macrobothriotaenia ficta (Cestoda: Proteocephalidea), a parasite of sunbeam snake (Xenopel- 
tis unicolor): example of convergent evolution. Zootaxa 3640: 485-499.

Scholz T., Kuchta R. 2017: A digest of fish tapeworms. Vie Milieu 67: 43-58.

Scholz T., Oros M. 2017: Caryophyllidea van Beneden in Carus, 1863. In: J.N. Caira and K. Jensen (Eds), Planetary Biodiversity Inventory (2008-2016): Tapeworms from Vertebrate Bowels of the Earth. The University of Kansas, Natural History Museum, Lawrence, USA, 25: 47-64.

Smyth J.D., McManus D.P. 1989: The Physiology and Biochemistry of Cestodes. Cambridge University Press, Cambridge, 398 pp.

WiLliams D.D. 1977: A key to caryophyllaeid cestodes of Wisconsin fishes. Iowa State J. Res. 51: 471-477.

Cite this article as: Oros M., Uhrovič D., Choudhury A., Mackiewicz J.S., Scholz T. 2020: Scolex morphology of monozoic tapeworms (Caryophyllidea) from the Nearctic Region: taxonomic and evolutionary implications. Folia Parasitol. 67: 003. 